\title{
Anabases
}

ANABASES Traditions et réceptions de l'Antiquité

$20 \mid 2014$

Varia

\section{Pour une traduction musicale des vers de Lucrèce}

\section{Guillaume Boussard}

\section{OpenEdition}

Journals

Édition électronique

URL : http://journals.openedition.org/anabases/5052

DOI : 10.4000/anabases.5052

ISSN : 2256-9421

\section{Éditeur}

E.R.A.S.M.E.

\section{Édition imprimée}

Date de publication : 1 novembre 2014

Pagination : 235-247

ISSN : 1774-4296

\section{Référence électronique}

Guillaume Boussard, «Pour une traduction musicale des vers de Lucrèce », Anabases [En ligne], 20 |

2014, mis en ligne le 01 novembre 2017, consulté le 21 octobre 2019. URL : http://

journals.openedition.org/anabases/5052 ; DOI : 10.4000/anabases.5052 


\title{
Pour une traduction musicale des vers de Lucrèce
}

Guillaume Boussard

\begin{abstract}
Comme le chien dont le flair découvre le gîte des fauves rôde-montagnes, tranquille, caché sous de vierges feuillages...

(Dē Nàtūiā Rērum, 1.404-405)
\end{abstract}

Dès le début du Chant 1, Lucrèce se place sous le double patronage poétique d'Homère et d'Ennius :

\section{Hommage}

1.120

...etsī praetereā tamen esse acherūsia templa

Ennius aeternīs expōnit uersibus ē dèns,

quō neque permaneant animae neque corpora nostra,

sed quaedam simulācra modîs pallentia mì̄is ;

unde sibi exortam semper flōrentis Homērì

1.125 commemorat speciem lacrimās effundere salsās

coepisse et rērum nātū ram expandere dictīs.

1.120 et pourtant, dans des vers immortels, Ennius nous explique qu'il existe, au bord de l'Achéron, des espaces où ne subsistent de nous ni les corps ni les âmes, mais comme des pâleurs, des façons de simulacres étranges ; il raconte avoir vu là-bas le visage d'Homère,

1.125 pour toujours florissant, qui versa des larmes salines en commençant à déployer la nature des choses. 
J'ai eu la chance de pratiquer à voix haute l'hexamètre français avec Philippe Brunet depuis 1997, et peu à peu l'envie de me frotter à cette métrique nouvelle a germé. J'ai commencé par Eschyle, en trasuisant Les Perses, avec Aymeric Münch et Yann Migoubert, puis Agamemnon seul. L'étape suivante fut le grand poème de Lucrèce, que je voulais rendre dans un français calibré pour la voix haute. En compagnie d'Emmanuel Lascoux, helléniste, latiniste et pianiste, nous construisons, depuis 2010, des récitals piano-voix chaque année renouvelés, qui permettent de " passer au gueuloir » un maximum de passages différents. Le très grand nombre de vers que ce doux travail nous a conduits à amender montre combien la perspective de représentations vivantes et publiques est une opportunité précieuse pour le traducteur. Ainsi, de très larges extraits ont été donnés sur scène depuis le début du travail en 2008, d'Argenton-sur-Creuse (Festival des Milliaires, à l'invitation de Véronique Pillon et Frédéric Billet) à Paris (Festival des Dionysies, avec la compagnie Démodocos). Placere, recommandaient les Anciens.

Que se passe-t-il quand on imite un Homère français en traduisant Lucrèce, ainsi que lui-même imitait le vieux maître pour décrire le monde ? Je commence toujours par dire à mi-voix, marmonner le vers latin avant de le traduire, pour en écouter d'abord le rythme et les sonorités : ce sont ces qualités-là qui seront premières pour l'auditeur à venir, elles précéderont, ne fût-ce qu'infimement, la compréhension du propos. Faisons le pari de donner des équivalents aux effets rythmiques, qu'ils soient évidents (retour du dactyle cinquième, catalexe) ou discrets, comme les génitifs à hiatus en -āī, ou les doubles élisions. Traduire à l'oreille devrait nous permettre, en outre, de nous prévenir du danger abyssal de la cacophonie, dont ne sut se garder Henri Clouard (Paris, 1935, traduction couronnée par l'Académie française), qui commençait ainsi sa version en prose du poème :

\section{$1.1 \quad$ Eneadum genetrix \\ Ô Mère d’Énée...}

Le lecteur croit entendre une interjection et une grossièreté avant le nom propre et se demande, navré, s'il ne s'est pas égaré dans quelque Énéide apocryphe dont les plaintes de Didon feraient le prologue.

Pourquoi Lucrèce a-t-il écrit le Dē Nātūrā Rèrum ? N'importe quel lecteur cultivé de son temps avait accès aux ouvres grecques d'Épicure ou de Démocrite : la diffusion de leurs doctrines n'avait pas besoin d'une simple redite. Même longtemps après, le plaisir presque pur du vers pris pour lui-même, pour sa valeur sonore toujours familière et nouvelle, suinte partout dans le Dé Nātūrā Rèrum, et nous supposons qu'il s'agit là du moteur principal de l'œuvre : constituer un ensemble immense de beaux hexamètres. De prime abord, le schéma métrique est une contrainte qui semble rétrécir, appauvrir le champ des possibles : le nez dans le guidon de l'alternance de thesis / arsis, on commence par tâtonner et compter sur ses doigts. Mais l'habitude de donner des vers en public accoutume l'aède à accueillir la variation et, assez rapidement, l'hexamètre se révèle une matrice féconde, gratifiant guide-plume qui offre une grande liberté et les variantes 
apparaissent pour ainsi dire d'elles-mêmes. À titre d'exemple, on comparera, pour le prologue du premier livre, deux états du texte dans lesquels je me reconnais autant :

\section{Propositions d'ouverture du Livre 1, vers 1-9}

1.1

1.5

1.5

1.5

\author{
Eneadum genetrix, hominum dìumque uoluptās, \\ alma Venus, caeti subter läbentia sìgna \\ quae mare nāuigerum, quae terrās frūgiferentīs \\ concelebrās, per tē quoniam genus omne animantum \\ concipitur ū̄sitque exortum lùmina sōlis, \\ tè, dea, tê fugiunt uentī, tè nübila caet \\ aduentumque tuum, tibi suāuis daedala tellūs \\ summittit flōrēs, tibi rìdent aequora pontī \\ plàcātumque nitet diffūsō lùmine caelum.
}

Alme Vénus, génitrice des fils d'Énéas, jouissance des humains et des dieux, sous les signes célestes qui passent, tu peuples tout dans la mer porte-nefs et les terres frugeuses : puisque c'est grâce à toi si toute espèce vivante se reproduit et parvient jusqu'à voir la lumière solaire, toi, ma Déesse, les vents te fuient, dans le ciel, les nuages fuient ton approche et pour toi la terre dédaléenne se parsème de fleurs, pour toi les flots batifolent et le ciel apaisé resplendit de fluide lumière!

Ou bien...

Plaisir des dieux et des hommes, faste Vénus qui fus mère des enfants d'Énéas, sous les signes glissants de la voûte, pour la mer porte-barque et les sols fructiferes, tu offres des réjouissances partout, puisque tout ce qui est doué d'âme vit et s'engendre par toi et par toi, aperçoit la lumière, les vents te fuient, ma déesse, et de même s'enfuient les nuages à ton approche, et pour toi les sols réinventent, suaves, toutes leurs fleurs et les vagues marines s'amusent, la voûte pacifiée dispense, profuse, des luminescences!

L'inventio a-t-elle sa place dans l'art de la traduction ? Les glissements légers du sens témoignent du brouillard de guerre où manœuvre fatalement l'interprète que deux mille ans séparent de la partition qu'il veut jouer. La contrainte prosodique fonctionne comme un catalyseur de l'inspiration et Lucrèce lui-même fait de la musicalité une dimension essentielle de son œuvre : 


\section{Le miel et l'absinthe (1.922-950)}

Nec mē animī fallit quam sint obscūra, sed ācrī percussit thyrsō laudis spēs magna meum cor et simul incussit suāuem mī in pectus amōrem Mūsārum, qūo nunc ìnstīnctus mente uigentī àuia Pìeridum perāgrō loca nūllius ante trìta solō. Iunat integrōs accēdere fontīs atque haurīre iuuatque noū̄s dēcerpere flō rēs insīgnemque meō capit̄i petere inde corōnam,

$1.930 \quad$ unde prius nūlla uè lārint tempora Mūsae; prīmum quod magnīs doceō dè rēbus et artīs relligiōnum animum nō dìs exsoluere pergōo, deinde quod obscūrā dè rē tam lūcida pangō carmina mūsaeō contingēns cūncta lepōre.

1.935 Id quoque enim nōn ab nüllā ratiōne uidētur; sed uelutī puerīs absinthia taetrā medentēs cum dare cōnantur, prius ōrās pōcula circum contingunt mellis dulc̄ flāuōque liquōre, ut puerōrum aetās inprōuida hüdificētur

1.940 lābrōrum tenus, intereā perpōtet amārum absinthī laticem dèceptaque nōn capiātur, sed potius tātà pactō recreàta ualèscat, sìc ego nunc, quoniam haec ratiō plèrumque uidètur trīstior esse quibus nōn est tractāta, retrōque

1.945 uolgus abhorret ab hāc, uolū̄ tibi suāuiloquentī carmine Pìeriō ratiōnem expōnere nostram et quasi mūsaeō dulcī contingere melle, sì tibi forte animum tātâ ratiōne tenēre uersibus in nostrīs possem, dum perspicis omnem

1.950 nātūram rērum, quā cōnstet cōmpta figùra.

Tout cela est bien sombre, je sais, mais le thyrse énergique frappe mon cour d'une grande espérance de gloire et mon torse s'emplit du même coup d'un tendre amour pour les Muses, 1.925 tendre amour qui occupe ma tête, à présent que je veille et parcours des espaces musiques sans route où personne n'a mis le pied. Quelle joie, d'atteindre des sources intactes, et d'y puiser! Quelle joie, d'y cueillir des fleurs insolites et de briguer pour mon front cette incomparable couronne 1.930 dont, avant moi, les Muses ne couronnèrent personne! 
Car mes paroles revêtent, d'abord, la plus haute importance : je dénoue les nœuds dont la religion nous entrave, et, de problèmes obscurs, moi, je compose un poème tout lumineux, comme imprégné de la grâce des Muses. Ce n'est pas sans raison que j'adopte une telle méthode : comme le médecin donne à boire à l'enfant de l'absinthe âcre - pour commencer, il enduit les rebords de la coupe avec du miel, en comptant sur la douce blondeur du breuvage pour que cet âge imprévoyant s'éjouisse les lèvres, mais qu'il avale d'un trait son amère coupe d'absinthe : le médecin ne veut pas le duper par cette manœuvre, mais, au contraire il veut que l'enfant recouvre ses forces je fais de même car notre doctrine parait, d'habitude, trop sévère aux gens qui ne l'adoptent pas, et la foule 1.945 s'en détourne, horrifiée, et du coup, j’ai voulu des paroles douces et dignes des muses Piérides, pour te la dire comme un poème enchanté par le miel de leurs voix musicales, si, par la grâce des vers, je peux installer ma doctrine dans ton esprit, afin que tu voies la nature des choses

1.950 tout entière et comment s'ordonne sa belle figure.

On ne peut traduire un poème qu'en cherchant à construire un poème, de préférence à la façon supposée des Anciens, c'est-à-dire à l'oreille, en ayant pour horizon la recitātiō, la lecture publique. On n'écrit pas de la même façon un texte destiné aux yeux et un poème fait pour l'oreille. Le retour inlassable des six cellules du vers latin crée d'abord une impression de très grande unité, impassible, dotée presque d'une volonté propre et semblant imposer telle ou telle formule, notamment en fin de vers.

Dans cet ensemble régulier, les " anomalies " crèvent de temps en temps la toile ; le traducteur, alors, doit chercher des équivalents à ces effets essentiels. On sait l'analyse que Pierre Fortassier ${ }^{24}$ fit des vers spondaïques; Lucrèce en use très peu (32 vers spondaïques pour 7415 vers en tout ${ }^{25}$ ), et leur rareté les rend d'autant plus expressifs. À titre d'exemple, voici le parti que j'en ai tiré dans le premier chant.

Tout est affaire de contexte : le spondée cinquième est parfois déplacé d'un vers ou deux dans la traduction, pourvu que la phrase reste frappée du même ralentissement, de la même claudication que la clausule latine.

24 P. ForTAsSier, Le spondä̈que expressif dans $l$ lliade et dans $l$ Odyssée, éd. Peeters, Paris \& Louvain, 1995.

25 S. Hellegourac'H, «Style et métrique dans l'œuvre de Lucrèce. Quelques observations ", Vita Latina, 130-131 (130), p. 7-17. 


\section{Vers spondaïques du livre 1}

\section{Précisions lexicales (1.58-61)}

quae nōs māteriem et genitālia corpora rēbus reddunda in ratiōne uocāre, et sēmina rērum appellāre suēmus, et haec eadem ūsurpāre corpora prìma, quod ex ilhis sunt omnia prìmīs.

Ces choses-la, nous aurons pour usage, dans notre analyse, de les nommer "matière " ou " corps générants ", ou " graines " et de leur attribuer le nom " corpuscules primaires " parce qu'ils sont les principes dont toute chose procède.

2. Horreur de la religion, éloge d'Épicure (1.62-65)

Hūmāna ante oculōs foedè cum ūita iacēret in terrīs oppressa graū sub relligiōne, quae caput à caeł regiōnibus ostendēbat horribili super aspectū mortālibus ìnstāns...

Comme la vie humaine gisait, indigne spectacle, contre terre, écrasée sous la religion oppressante qui, du haut des régions du ciel, exhibait sa face en planant au-dessus des mortels, vision terrifiante...

\section{Nécessité de la loi naturelle (1.584-586)}

Dènique iam quoniam generātim reddita finis crēscendī rēbus cōnstat ū̄tamque tenendī, et quid quaeque queant per foedera nātū rāī, quid porrō nequeant, sancītum quandoquidem extat...

Bref. Pour chaque génération, une borne limite

1.585 la croissance des corps et leur durée d'existence, ce que les lois naturelles permettent, et ce qu'au contraire elles prohibent dans le cadre de lois précises : 
4. Nécessité d'un minimum de matière (1.615-618)

1.615

Praetereā nisi erit minimum, paruissima quaeque corpora cōnstābunt ex partibus ìnfiñ̄itis, quippe ubi dimidiae partis pars semper habēbit dimidiam partem nec rēs praefiniet ülla.

1.615

S'il n'est aucun minimum, la chose la plus minuscule sera faite d'une infinité de parties d'elle-même, toute moitié de moitié aura sa moitié elle-même sans que jamais la division ne trouve un terme.

\section{Nécessité de l'infinité de l'espace (1.985-991)}

nec foret omnīno caelum neque lümina sōlis, quippe ubi māteriēs omnis cumulāta iacēret ex infinītō iam tempore subsìdendō.

il n'existerait plus ni ciel ni lumière solaire

1.990 puisque la matière s'amasserait toute entière et resterait indéfiniment immobile, inerte.

6. Nécessité de l'opposition matière-vide (1.1077-1080)

Nec quisquam locus est, quō corpora cum vēnēre, ponderis àmissā ū possint stāre in inānī ; nec quod ināne autem est ùlti subsistere dēbet,

1.1080 quīn, sua quod nātūra petit, concēere pergat.

Car il n'existe aucun lieu dans lequel les objets se trouvent privés de poids, aucun lieu où ils puissent tenir dans le vide; et le vide ne peut se trouver nulle part sous un être

1.1080 sans lui céder la place car sa nature l'exige.

\section{Immensité des perspectives scientifiques (1.1114-1117, final)}

1.1115

Haec sīc pernōscēs paruā perductus opellā; namque alid ex aliō clārescet nec tibi caeca nox iter è ripiet, qū̄n ultima nātū rāì peruideās : ita rēs accendent lümina rēbus. 
Tu parviendras à savoir tout cela sans trop de peine,

1.1115 une idée éclairera l'autre, et la nuit aveuglante ne te voilera pas le chemin des recoins ultimes de la nature : les choses mettront en lumière les choses.

Mes propres difficultés à lire les vers latins à voix haute m'ont mis la puce à l'oreille. Ainsi, en improvisant la scansion d'un vers latin, le lecteur d'aujourd'hui peut être déconcerté par les élisions, surtout quand elles frappent à la fois l'attaque et la finale d'un même mot. Ainsi, par exemple, chez Catulle :

\subsection{Vivāmus, mea Lesbia atque amēmus}

où la conjonction atque conjugue le nom de Lesbie au verbe aimer. Ce procédé est-il expressif chez Lucrèce ? S'il l'est, peut-on lui trouver des équivalents ? En première hypothèse, il ne serait guère étonnant que la double élision n'ait rien de fortuit dans le Dē Nâtūrā Rērum : toute la physique épicurienne consiste en une théorie de la jonction et de la disjonction des atomes, explicitement comparés aux lettres de l'alphabet :

\section{Alphabet atomique}

$$
\begin{aligned}
& \text {...atque eadem magnī rēfert prìmōrdia saepe } \\
& \text { cum quibus et quā } \bar{a} \text { positū rā contineantur } \\
& \text { et quōs inter sē dent mōtūs accipiantque; } \\
& \text { namque eadem caelum mare terrās flümina sōlem } \\
& \text { cōnstituunt, eadem frūgēs arbustā animantīs, } \\
& \text { uērum alīis aliōque modō commixta mouentur. } \\
& \text { Quīn etiam passim nostrīs in uersibus ipsīs } \\
& \text { multa elementa uidès multīs commūnia uerbīs, } \\
& \text { cum tamen inter sē uersūs àc uerba necessest } \\
& \text { cōnfiteāre et rēe et sonitū distā re sonantī. } \\
& \text { Tantum elementa queunt permū tā tō ōrdine sōtō; } \\
& \text { at rērum quae sunt prīmōrdia, plūra adhibēre } \\
& \text { possunt unde queant uariae rēs quaeque creārī. }
\end{aligned}
$$

...et l'important, concernant les atomes, ce sont les manières dont ils se mêlent entre eux, avec quoi et comment ils s'agencent, quels mouvements ils se donnent, quels mouvements ils reçoivent ;

1.820 ciel, terre et mer, soleil et fleuves sont faits de principes, qui font aussi les arbustes, les fruits, les espèces vivantes : ce sont leurs combinaisons et leurs mouvements qui different. Plus fort encore : les lettres, nombreuses dans nos hexamètres, 
sont les facteurs communs à l'ensemble des mots innombrables, et pourtant, les mots et les vers, il faut bien reconnaitre qu'ils different entre eux par le sens et le son qu'ils produisent : l'ordre des lettres permet à lui seul un tel phénomène, et les atomes peuvent se combiner de manières encore plus nombreuses, pour faire les choses diverses.

L'expression "anima atque animus » fournit des éléments à l'enquête. Sa première occurrence dans le poème est précisément l'endroit où l'indissociabilité de l'âme et de l'esprit est proclamée. La conséquence métaphysique de cette donnée physique est bien entendue décisive, puisqu'il s'agit de montrer que rien ne survit après que l'homme est mort :

\section{L'âme-esprit : jonction et disjonction}

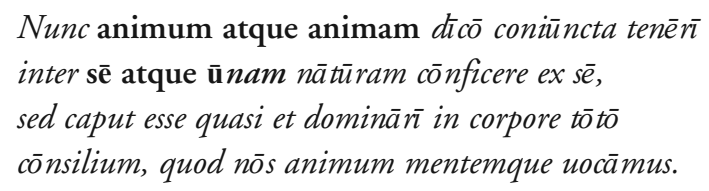
ses éléments ne forment à deux qu'une seule substance mais ce qui mène le corps, un peu comme la tête, on l'appelle, siège de la pensée, " esprit " ou " intelligence ".

De manière frappante, la double élision apparaît dans deux vers consécutifs : nous avons choisi de rendre ce jeu par la création du mot âme-esprit. La langue latine forge volontiers des mots composés (ceux de Lucrèce ont été amplement étudiés, notamment par L. $\mathrm{Nadjo}^{26}$ ) et nous supposons que, quand elle est doublement élidée, la conjonction atque peut jouer un rôle analogue au tiret dans notre mode de composition lexicale. L'hendiadyn convoque d'ailleurs volontiers la double élision d'atque chez Virgile :

Tum Danā̄, gemitū atque è reptae virginis ì $r \bar{a} . .$.

(Énéide, II, 413)

L'observateur, amusé, constate que Virgile reprend l'hendiadyn avec double élision, mais disloque les deux termes gemitu et ira. Ainsi, la variation souligne l'hommage.

26 L. NAdjo, La composition nominale : Études de linguistique latine, L'Harmattan, Paris, 2010 . 
Rendre par une création lexicale un fait prosodique est risqué, mais le contexte ici semble l'autoriser, de même que les trois hiatus du vers français 3.139 nous semblent ici permis s'ils fournissent l'écho inversé des doubles élisions du passage. Cependant, cette "seule substance " est constituée de deux substances différentes, l'une féminine et l'autre masculine. Aussi, prudemment, nous n'avons écrit âme-esprit qu'en face des passages où Lucrèce emploie l'expression anima atque animus, et nous avons dissocié « l'esprit » de « l'âme » dans tous les autres cas. Le néologisme est l'un des atouts revendiqués du poète :

\section{Néologismes}

1.140

Nec mè animī fallit Grāiōrum obscūra reperta difficile inlustrāre latīn̄s uersibus esse, multa noūis uerbīs praesertim cum sit agendum propter egestātem linguae et rērum nouitātem; sed tua mē uirtūs tamen et spērāta uoluptās suāuis amīcitiae quemūis efferre labō rem suādet et indūcit noctēs vigilāre serēnās quaerentem dictīs quibus et quō carmine dèmum clāra tuae possim praepandere lümina mentī, 1.145 rēs quibus occultās penitus conūisere possīs.

Il ne m’a pas échappé que mettre en lumière ces sombres découvertes des Grecs, par des vers latins n'est pas simple : leur traduction exige beaucoup de néologismes tant notre langue est pauvre et la matière, nouvelle ;

1.140 mais, malgré tout, ta valeur et le plaisir que j'espère en ta douce amitié me font surmonter toute peine et m'invitent à chercher, dans le calme nocturne où je veille, les mots ou plus exactement le poème qui déploierait devant ton esprit d'éclatantes lumières

1.145 et te dévoilerait le secret de toutes les choses.

À l'autre bout de la chaîne, du côté de l'expression de la disjonction, le lecteurauditeur de Lucrèce remarque la concurrence des génitifs en -ae et des génitifs " archaïques " en $-\bar{a} \bar{l}$, dont on trouve un exemple saisissant dans les vers qui suivent presque immédiatement ceux que nous citions tout-à-l'heure : 


\section{L'âme-esprit : jonction et disjonction (suite)}

Cētera pars animae per tōtum dissita corpus

pāret et ad nümen mentis mōmenque mouetur.

3.145 Idque sibì sōlum per sē sapit et sibi gaudet,

cum neque rēs animam neque corpus commouet ūna.

Et quasi, cum caput aut oculus temptante dolōre

laeditur in nōbīs, nōn omnī concruciāmur

corpore, sīc animus nonnumquam laeditur ipse

3.150 laetitiāque uiget, cum cētera pars animāì

per membra atque artūs nūllā nouitāte ciētur;

L'autre part - l'âme - épandue dans le corps tout entier, suit les ordres, les impulsions et les règles que dicte l'intelligence

3.145 car l'esprit seul peut savoir et se réjouir de lui-même, même quand nulle chose n'émeut l'organisme ni l'âme.

C'est comme quand on souffre des yeux, qu'on a mal à la tête, ce n'est pas tout l'organisme qui subit la souffrance, de la même façon, parfois, l'esprit souffre ou jubile, se revigore de joie, mais l'autre part d'âme n'éprouve à travers corps et membres, rien de nouveau qui l'émeuve.

Le génitif en diphtongue - ae du vers 3.143 se trouve dans un contexte où le poète insiste sur l'union des deux substances, tandis que le génitif à hiatus du vers 3.150 souligne leur indépendance. Nous essayons de rendre ce jeu par l'écho des expressions "L'autre part - l'âme - " / "l'autre part d'âme " placées en tête puis en fin de vers. L'hendiadyn per membra atque artūs nous semble devoir être traduit par une expression des plus ramassées : "À travers corps et membres » est possible.

Chez les épicuriens, le monde des atomes est le théâtre de cette tension entre jonction et disjonction des substances ; un peu plus loin, dans ce passage capital, le jeu de concaténation par élision se resserre spectaculairement au vers 3.159, évoque en neuf

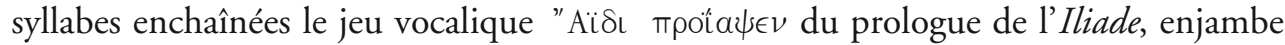
allègrement les vers 3.163-164 et culmine au quod erat dèmōnstrandum du vers 3.167.

\section{L'âme-esprit : jonction et disjonction (fin)}

Dēnique concidere ex animì terrōre uidèmus saepe hominēs; facile ut quīū̄s hinc nōscere possit esse animam cum animō conī̄ nctam, quae cum animī [ū]] 
3.160

percussa est, exim corpus prōpellit et $\bar{c}$ cit.

Haec eadem ratiō nātū ram anim̄̄ atque animā̄

corpoream docet esse; ubi enim prōpellere membra,

corripere ex somnō corpus mūtā reque uultum

atque hominem tōtum regere ac uersāre uidētur,

3.165

quōrum nīl fierī sine tāctū posse uidēmus

nec tāctum porrō sine corpore, nōnne fatendumst

corporeā nātū rā animum cōnstāre animamque?

Bref. Bien souvent, les hommes succombent devant la panique de leur esprit, et cela nous permet de comprendre sans peine que l'esprit et l'âme sont joints l'un à l'autre : quand l'âme

3.160 est frappée par l'esprit, elle frappe le corps et l'excite.

Cela explique pourquoi l'âme-esprit est fait de substance corporelle : en effet, chaque fois qu'il nous bouge les membres et les arrache au sommeil, le corps modifie son allure, semble régner sur l'être en entier, et le mettre aux tortures, or, sans le sens tactile, c'est évidemment impossible et, sans le corps, il n'est pas de toucher : ne doit-on reconnaître que l'esprit et l'âme sont corporels par nature ?

Tel est donc le projet qui sous-tend notre traduction de Lucrèce : proposer un texte qui soit à Lucrèce l'équivalent de l'Élégie à Marienbad de Goethe par Jean Tardieu, ou du Catulle d'André Markowicz ou de l'Homère de Philippe Brunet car aucune œuvre n'existe jamais seule mais les poèmes se reprennent, s'imitent, rivalisent, et le traducteur commettrait une lourde erreur en négligeant la tradition agonistique qui entoure toute poésie ancienne. On rêve de disposer de l'ensemble de la poésie ancienne traduite selon des principes musicaux. Après Eschyle, Aymeric Münch s'est lancé chez Virgile, Ennius attend son interprète... Ars longa.

Guillaume BOUSSARD

Centre de poésie et de théatre antiques
Compagnie Démodocos
Route des Méandres
75530 Mauny
guillaume.boussard@laposte.net




\section{Éditions et traductions françaises remarquables}

(1473) Editio princeps, T. FerRANDUs, Brescia.

(1650) Lucrèce, De la nature des choses, Abbé de Marolles, Paris [première traduction complète en français, en prose].

1823 Lucrèce, De la nature des choses, J.-B. de Pongerville, Paris [première traduction complète en français versifié : des alexandrins].

1829 Lucrèce, De la nature des choses, J.-B. de Pongerville, Paris [en prose].

1920 Lucrèce, De la Nature, A. Ernout, Les Belles-Lettres, C.U.F., Paris [en prose].

1931 Lucrèce, De la nature, H. Clouard, Classiques Garnier, Paris [en prose].

1954 Lucrèce, De la nature, R. WaLTZ, Les Belles-Lettres, Paris [en prose].

1993 Lucrèce, De la nature, J. Kany-Turpin, Aubier, Paris [en vers libres].

2000 Lucrèce, De la nature, Ch. GuITARD, Imprimerie Nationale [en prose].

2002 Lucrèce, De la nature des choses, B. Pautrat, Le Livre de Poche, Paris [en alexandrins].

2004 Lucrèce, De la nature des choses, Ch. LABRE, Arléa, Paris [en prose].

2010 Lucrèce, La Nature des choses, J. PigeAud, in Les Épicuriens, Bibliothèque de la Pléiade, Gallimard, Paris [en vers libres].

2012 Lucrèce, De la nature, O. Sers, Les Belles-Lettres, Paris [en alexandrins].

\section{Récitals en hexamètres}

2009 Hymne à la matière, extraits des deux premiers chants du De Natura Rerum de Lucrèce, par Guillaume Boussard et le groupe néo-folk Battan l'Otto, au festival des Dionysies (Paris).

Lecture d'extraits du chant 1 par Guillaume Boussard au festival des Milliaires (Argenton-sur-Creuse).

2010 Momentum Lucretianum, récital d'Emmanuel Lascoux (piano, voix) et Guillaume Boussard (voix), extraits des trois premiers chants de Lucrèce. Dionysies puis Maison de l'Europe (Paris).

2011 Le Rythme des Choses, récital d'Emmanuel Lascoux et Guillaume Boussard, extraits des trois premiers chants de Lucrèce. Dionysies puis E.N.S. (Paris).

2012 Lucrèce ou l'exact maître, récital d'Emmanuel Lascoux et Guillaume Boussard, extraits des quatre premiers chants de Lucrèce. Dionysies puis E.N.S., puis Cerisy-la-Salle (colloque Le moment du vivant).

2013 Lucrèce, récital d'Emmanuel Lascoux et Guillaume Boussard, extraits des quatre premiers chants de Lucrèce. Dionysies puis Milliaires. 